\title{
Raman spectrum of plutonium dioxide: Vibrational and crystal field modes
}

\author{
M. Naji, ${ }^{1}$ N. Magnani, ${ }^{1}$ L. J. Bonales,${ }^{2}$ S. Mastromarino, ${ }^{1,3}$ J.-Y. Colle,${ }^{1}$ J. Cobos,${ }^{2}$ and D. Manara ${ }^{1, *}$ \\ ${ }^{1}$ European Commission, DG Joint Research Centre-JRC, Directorate G - Nuclear Safety and Security, \\ Postfach 2340, D-76125 Karlsruhe, Germany \\ ${ }^{2}$ Centro de Investigaciones Energéticas, Medioambientales y Tecnológicas, CIEMAT Avenida Complutense, 40, 28040 Madrid, Spain \\ ${ }^{3}$ Università degli Studi di Roma "La Sapienza", Piazzale Aldo Moro 5, 00185 Rome, Italy
}

(Received 25 November 2015; revised manuscript received 25 January 2017; published 29 March 2017)

\begin{abstract}
The Raman spectrum of plutonium dioxide is studied both experimentally and theoretically. Particular attention has been devoted to the identification of high-energy modes at 2110 and $2620 \mathrm{~cm}^{-1}$, whose attribution has so far been controversial. The temperature dependence of both modes suggests an electronic origin for them. Original crystal field (CF) calculations reported in this work show that these two modes can be respectively assigned to the $\Gamma_{1} \rightarrow \Gamma_{5}$ and $\Gamma_{1} \rightarrow \Gamma_{3} \mathrm{CF}$ transitions within the ${ }^{5} I_{4}$ manifold. These two modes, together with the only vibrational line foreseen by the group theory for the $\mathrm{Fm}-3 m \mathrm{PuO}_{2}$ symmetry - the $T_{2 g} \mathrm{Pu}-\mathrm{O}$ stretching mode observed at $478 \mathrm{~cm}^{-1}$ — can thus be used as a Raman fingerprint of fcc plutonium dioxide.
\end{abstract}

DOI: 10.1103/PhysRevB.95.104307

\section{INTRODUCTION}

Plutonium dioxide spectroscopy has important applications in nuclear waste management, nuclear forensic, and safeguard activities [1,2]. In particular, the Raman spectrum of plutonium dioxide $\left(\mathrm{PuO}_{2}\right)$ has been studied in a few research laboratories in the last couple of decades [3-6]. Plutonium dioxide crystallizes in the fcc fluoritelike structure, belonging to the $F m-3 m$ space group. The factor group theory at the $\Gamma$ point for this type of crystal symmetry was fully derived by Shimanouchi et al. [7]. It foresees only one triply degenerate $T_{2 g}$ Raman active vibrational mode, and one infrared active $T_{1 u}$ mode at a lower energy. Since the work by Shimanouchi et al., these modes have been experimentally observed for various actinide dioxides and other fluoritelike materials [8-11], including plutonium dioxide $[4,5,12]$. However, the Raman spectrum of plutonium dioxide was reported up to higher Raman shift energies (beyond $1500 \mathrm{~cm}^{-1}$ ) only recently [12]. A few crucial features of the high-energy part of the $\mathrm{PuO}_{2}$ Raman spectrum remain to-date unclarified, which has not permitted a satisfactory definition of a precise Raman fingerprint for $\mathrm{PuO}_{2}$. Among those features, the most remarkable are two strong peaks consistently observed by Sarsfield et al. [12] at $2110 \pm 10$ and $2620 \pm 10 \mathrm{~cm}^{-1}$. Despite some attempts, the nature of these two lines could not be clearly defined. Sarsfield et al. [12] analyzed in depth various possible attributions for them, both in terms of higher-order phonon combinations and surface impurity modes, but could not find any satisfactory assignment. Finally, these authors suggested that these lines might have an electronic origin.

In this respect, there is a vast literature discussing the crystal field (CF) potential of $\mathrm{UO}_{2}$ and $\mathrm{NpO}_{2}$, particularly in relation to their peculiar low-temperature magnetic properties [13]. Less attention has been devoted to $\mathrm{PuO}_{2}$, mainly because, at a

\footnotetext{
*Corresponding author: dario.manara@ec.europa.eu

Published by the American Physical Society under the terms of the Creative Commons Attribution 4.0 International license. Further distribution of this work must maintain attribution to the author(s) and the published article's title, journal citation, and DOI.
}

first sight, its temperature-independent magnetic susceptibility [14] is compatible with its $\Gamma_{1}$ singlet ground state. This is expected from the simple argument that the $\mathrm{CF}$ states for $\mathrm{Pu}^{4+}$ ions in a given ligand geometry should roughly be the inverse as those for $\mathrm{U}^{4+}$ [15]. However, this single-ion picture turns out to be problematic upon further inspection, in particular because the energy gap between the two lowest CF levels detected by inelastic neutron scattering (INS) is much smaller than the value which was estimated from magnetic measurements (in order to maintain a constant paramagnetic susceptibility up to $1000 \mathrm{~K}$ ); this issue is still a debated problem today [16]. It must be remarked that INS is only sensitive to the $\Gamma_{1} \rightarrow$ $\Gamma_{4}$ transition, and a complete spectroscopic investigation of the lowest ${ }^{5} I_{4}$ manifold is still lacking and would be highly desirable in order to test it against current theories.

Experimentally, in addition to INS, electronic Raman scattering (ERS) played a crucial role to determine the various split LSJ manifolds of the $4,5 f$ ions [17]. In $\mathrm{UO}_{2}$, ERS elucidate the manifestation of the strong electron-phonon interactions in the resonant coupling between $n \mathrm{LO}(n=1-4)$ phonons and $3 H_{4}^{\prime} \rightarrow 3 F_{2}^{\prime} \mathrm{U}^{4+}$ intermultiplet $\mathrm{CF}$ excitations [18]. Consequently, one would expect that the Raman effect would be of some interest in the study of $\mathrm{CF}$ transitions in $\mathrm{PuO}_{2}$.

The experimental part of this study was carried out with the help of an original encapsulation technique, described in a very recent publication [19]. Among other things, this technique permits, for the investigation of such highly radioactive materials, the use of different excitation laser sources, and the employment of the triple subtractive spectrometer configuration. The first feature is important for the analysis of resonant and energy dispersive modes and for the detection of fluorescence features in the Raman spectra; the second is necessary for the study of low-energy Raman lines and anti-Stokes peaks. In particular, the study of the $T_{2 g}$ antiStokes band, carried out in this research on $\mathrm{PuO}_{2}$ in parallel to the Stokes spectrum, allowed us to reasonably estimate a temperature dependence of the observed Raman modes, especially the above-mentioned high-energy bands. Moreover, $\mathrm{CF}$ calculations performed in the present research soundly support the attribution of these high-energy lines to $\mathrm{PuO}_{2} \mathrm{CF}$ transitions. Such a conclusion, together with the already sound theoretical and experimental description of the vibrational part 
of the Raman spectrum, allows us to propose a consistent Raman fingerprint for plutonium dioxide.

\section{EXPERIMENTAL METHODS}

\section{A. Samples}

The current Raman spectroscopy measurements were performed on a solid polycrystalline $\mathrm{PuO}_{2}$ disk, prepared following the procedure described by De Bruycker et al. [20]. The starting material was based on beads obtained by gelsupported precipitation (SOL-GEL). A disk-shaped sample of 8-9 $\mathrm{mm}$ in diameter and about $1 \mathrm{~mm}$ in thickness was obtained using a bidirectional press. The samples were then sintered in an atmosphere of $\mathrm{Ar}+\mathrm{H}_{2}$ with $1500 \mathrm{ppm}$ of $\mathrm{H}_{2} \mathrm{O}$ to obtain dense material. In order to obtain stoichiometric material $(O / M=2.00)$ and remove the defects produced during the fabrication, the $\mathrm{PuO}_{2}$ sample was subjected to two consecutive heat treatments under a flow of air at $1423 \mathrm{~K}$ for $8 \mathrm{~h}$. Already after the first heat treatment the measured weight gain of the sample corresponded to stoichiometric $\mathrm{PuO}_{2}$ separately measured by thermogravimetry (TG). Since, in addition, no further change was noticed after annealing the samples for a second time, it was considered that $\mathrm{PuO}_{2.00}$ stoichiometry had been reached. A lattice parameter of $0.5396(1) \AA$ was measured by $\mathrm{x}$-ray diffraction (XRD), corresponding to the literature value for $\mathrm{PuO}_{2}$ [21]. The average crystallite size measured by XRD and estimated with the help of scanning electron microscopy (SEM) on a polished surface was (5 \pm 3$) \mu \mathrm{m}$.

The isotopic composition of the plutonium employed was checked by high resolution Gamma spectroscopy (HRGS) to be 93.4 wt. $\%{ }^{239} \mathrm{Pu}$ and $6.4 \mathrm{wt} . \%{ }^{240} \mathrm{Pu}$. Traces $(<0.2 \mathrm{wt} . \%)$ of ${ }^{241} \mathrm{Pu}$ and ${ }^{241} \mathrm{Am}$ stemming from $\beta$ decay of ${ }^{241} \mathrm{Pu}$ were detected by thermal ionization mass spectrometry (TIMS).

\section{B. Raman measurements}

A smaller fragment (about $5 \times 1 \times 1) \mathrm{mm}^{3}$ of the $\mathrm{PuO}_{2}$ sample was encapsulated in an $\alpha$-shielding Plexiglas container equipped with a quartz window through which micro-Raman spectra were recorded. The whole procedure has been described in detail elsewhere [19]. The Raman microscope was equipped for the present measurements with a long working distance $(10.6 \mathrm{~mm})$ objective, which offers a 0.5 numerical aperture with $\times 50$ magnification. The Raman spectrometer employed in this research is a Jobin-Yvon T 64000 equipped with a 1800 grooves per mm grating, a low noise liquid nitrogen cooled symphony $\mathrm{CCD}$ detector, and a subtractive premonochromator (in triple mode) which allows access to anti-Stokes spectrum lines while blocking the elastic Rayleigh line. Excitation sources used in this work are the $488 \mathrm{~nm}(2.53 \mathrm{eV})$ or $514.5 \mathrm{~nm}(2.41 \mathrm{eV})$ lines of an $\mathrm{Ar}^{+}$Coherent ${ }^{\circledR}$ continuous wave $(\mathrm{CW})$ laser or the $647 \mathrm{~nm}$ $(1.91 \mathrm{eV})$ or $752 \mathrm{~nm}(1.65 \mathrm{eV})$ lines of a similar $\mathrm{Kr}^{+}$laser. Both laser sources have a controllable nominal power up to $1.5 \mathrm{~W}$. Typical laser power values at the sample surface ranged between 5 and $300 \mathrm{~mW}$, whereby the highest power values were used in order to heat the sample in situ. The power impinging the sample surface was measured by a Coherent ${ }^{\circledR}$ power meter placed at a position corresponding to the sample surface. It is lower by approximately a factor 5 than the actual power at the exit of the laser cavity. Using the long focal $\times 50$ objective and the single spectrometer mode permits a good spectral resolution $\left( \pm 1 \mathrm{~cm}^{-1}\right)$ independently of the surface shape, with a spatial resolution of $2 \mu \mathrm{m} \times 2 \mu \mathrm{m}$ on the sample surface. The spectrograph is calibrated with the $T_{2 g}$ excitation of a silicon single crystal, set at $520.5 \mathrm{~cm}^{-1}$ [22]. The instrument is calibrated on a daily basis prior to measurements.

\section{Temperature determination}

For Raman laser heating experiments, the Raman laser was used as an excitation and heating source. The laser power was then increased stepwise and the Stokes/anti-Stokes (SAS) Raman spectra were collected at different powers.

The absolute temperature $\left(T_{\mathrm{SAS}}\right)$ was derived from the ratio $\left(R_{\mathrm{SAS}}\right)$ between the experimental intensities of the anti-Stokes and Stokes $T_{2 g}$ peaks according to Bose-Einstein statistics. Prior to these calculations, Stokes and anti-Stokes Raman spectra were corrected for the instrumental response according to the procedure described elsewhere [23,24]. Despite the instrumental correction, the value of $T_{\mathrm{SAS}}$ can represent the real sample temperature in a rather inaccurate fashion, due to uncontrollable sample-dependent factors affecting the intensities of the Stokes and anti-Stokes lines, such as impurity fluorescence, roughness scattering, etc. Nonetheless, the values obtained through Stokes and anti-Stokes are sufficient to perform a qualitative study of the temperature evolution of the Raman lines observed in this work.

To check the accuracy of the SAS method, a $\mathrm{CeO}_{2}$ sample was heated in a Linkam TS 1500 device and the Raman spectra (SAS part) were recorded. The absolute temperatures (calculated from SAS ratio) were compared to those of the Linkam device. Both temperatures converge to almost the same values for low laser power/temperature; however, as the laser power/temperature increases $(T>750 \mathrm{~K})$, the temperatures values diverge. The temperature uncertainty was calculated according to the method described by LaPlant et al. [25]. Note that the theoretical error bars on temperatures (as determined from this method) are comparable to those obtained experimentally on the $\mathrm{CeO}_{2}$ sample, thus giving reasonable $\Delta T$ values between $\sim 7$ and $20 \mathrm{~K}$ (for $T<750 \mathrm{~K}$ ).

\section{RESULTS AND DISCUSSION}

\section{A. Raman spectra of $\mathrm{PuO}_{2}$ with different excitation sources}

Figure 1 shows the Stokes Raman spectra measured on $\mathrm{PuO}_{2}$ with the four excitation sources used in this research. The acquisition of Raman spectra recorded at 752 and $647 \mathrm{~nm}$ laser excitations had to be cut well below $3000 \mathrm{~cm}^{-1}$ because the spectral limit of the CCD detector was reached. Some peaks are clearly observable, which are summarized in Table I.

The $T_{2 g}$ peak at $(478 \pm 2) \mathrm{cm}^{-1}$ dominates the spectra recorded with all the excitation sources. A weaker mode is observable in all the spectra at $\sim 578 \mathrm{~cm}^{-1}$. This mode has been assigned to the $T_{1 u} \mathrm{LO} 2$ phonon at the $\Gamma$ point of the Brillouin zone, in agreement with the density functional theory (DFT) calculations performed by Zhang et al. [26] and an original many-body approach proposed by Yin and Savrasov [27]. Such vibrational mode would be Raman forbidden in a perfect 


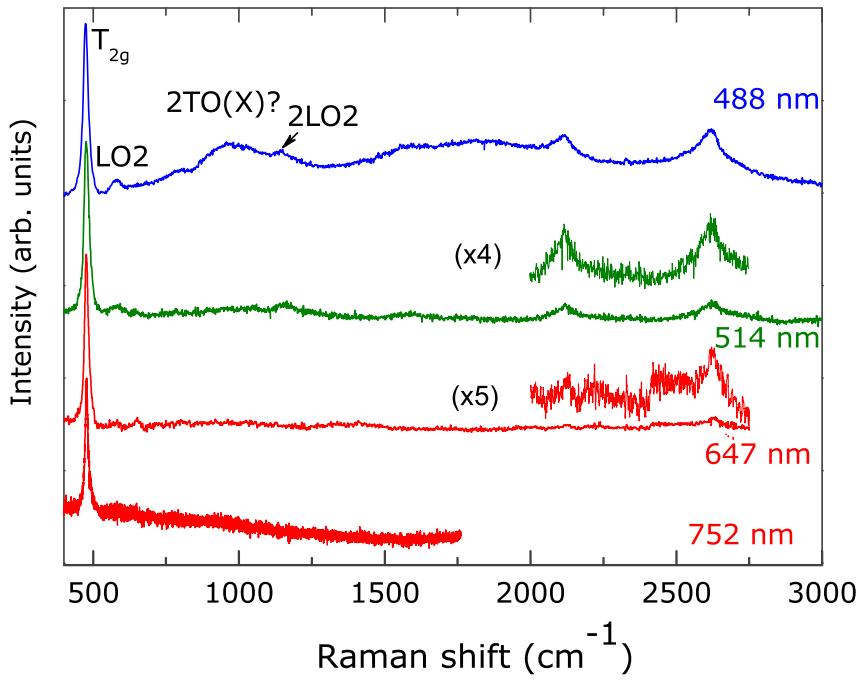

FIG. 1. Spectra of $\mathrm{PuO}_{2}$ acquired with different laser energies $488 \mathrm{~nm}(2.53 \mathrm{eV}), 514.5 \mathrm{~nm}(2.41 \mathrm{eV}), 647 \mathrm{~nm}(1.91 \mathrm{eV})$, and $752 \mathrm{~nm}(1.65 \mathrm{eV})$. All bands are assigned in accordance with Table I.

Fm-3m crystal. It is activated by Frenkel-type oxygen defects, as suggested in recent literature for an analogous mode predicted and observed in $\mathrm{UO}_{2}$ [28-30]. The first overtone (2LO2) of this latter line can be observed at $1156 \mathrm{~cm}^{-1}$ in the spectra excited with the higher energy sources, 2.41 and $2.53 \mathrm{eV}$. The fact that the $2 \mathrm{LO} 2$ line is only visible at higher excitation energies suggests that in $\mathrm{PuO}_{2}$, like in $\mathrm{UO}_{2}$, the mode is activated via a resonance multiphonon process, its absorption edge lying between 2 and $2.4 \mathrm{eV}$. The deconvolution of this line is difficult in the 488-nm spectrum because it overlaps with a further broad band centered at $956 \mathrm{~cm}^{-1}$. This band might be similar to the weak one observed by Livneh and Sterer in $\mathrm{UO}_{2}$ [10], and assigned to disorder-activated 2TO2 phonon at the Brillouin zone's $X$ edge. In fact, this band is centered at exactly twice the energy of the $T_{2 g}$ band, whereas its larger width can be attributed to a shortening of the phonon lifetime observable in the overtone. A similar behaviour is observed in the $2 \mathrm{LO} 2$ band centered at $1156 \mathrm{~cm}^{-1}$.

Other broad bands can be seen in the 488-nm and the 514-nm spectra between 1500 and $2000 \mathrm{~cm}^{-1}$. These

TABLE I. Attribution of the Raman band position for $\mathrm{PuO}_{2}$.

\begin{tabular}{lll}
\hline \hline Raman band position $\left(\mathrm{cm}^{-1}\right)$ & Assignment & Reference \\
\hline $478 \pm 2$ & $T_{2 g}$ & {$[12]$} \\
575 & $\mathrm{LO} 2$ & {$[12]$} \\
956 & $2 \mathrm{TO} 2(X) ?$ & This work \\
1156 & $2 \mathrm{LO} 2$ & {$[12]$} \\
1531 & $2 \mathrm{LO} 1+\mathrm{LO} 2$ & This work \\
1555 & $\mathrm{O}_{2}$ & {$[31]$} \\
1600 & $s p^{2}(\mathrm{C}=\mathrm{C})$ & {$[32]$} \\
$1800^{\mathrm{a}}$ & Fluorescence & This work \\
$2110^{\mathrm{b}}$ & $\Gamma_{1} \rightarrow \Gamma_{5}(\mathrm{CF})$ & This work \\
$2620^{\mathrm{b}}$ & $\Gamma_{1} \rightarrow \Gamma_{3}(\mathrm{CF})$ & This work \\
\hline \hline
\end{tabular}

${ }^{a}$ Visible only with the $488 \mathrm{~nm}$ laser line.

${ }^{\mathrm{b}}$ Not observed with the $752 \mathrm{~nm}$ laser line. can be attributed to further overtones (e.g., $2 \mathrm{LO} 1+\mathrm{LO} 2=$ $\left.1531 \mathrm{~cm}^{-1}\right)$, adsorbed $\mathrm{O}_{2}$ molecules $\left(1555 \mathrm{~cm}^{-1}\right)$ [31], a slight carbon contamination (near $1600 \mathrm{~cm}^{-1}$ ) [32], and fluorescence effects (broad band centered $1800 \mathrm{~cm}^{-1}$ in the $488-\mathrm{nm}$ spectrum only). The weak carbon and adsorbed oxygen lines becomes more visible at higher excitation energies due to the absolute frequency dependence of the Raman cross section. Moreover, the attribution to overtones of bands centered at spectral positions corresponding to integer multiples of known modes is consistent with the fact that they are only observable with higher energy excitation sources.

Beyond $2000 \mathrm{~cm}^{-1}$ only three excitation sources could be used, because the $752 \mathrm{~nm}$ laser line is too close to the high-wavelength spectral edge of the spectrometer. In the three cases, two peaks can be obviously observed at 2110 and $2620 \mathrm{~cm}^{-1}$, though less easily in the 647-nm spectrum. These peaks were already observed and studied by Sarsfield et al. [12], although no satisfactory attribution was found for them. The only possibility left open by Sarsfield et al. was indeed that those peaks have an electronic origin, a hypothesis which we are further testing in the present work.

It can be noticed, incidentally, that a photon energy dependence of the intensity of these two bands is most likely to be present also in this case. However, such an observation should be treated with care, because no obvious photon-energy dependence was observed by Sarsfield et al. with other experimental facilities and excitation sources for the $2620 \mathrm{~cm}^{-1}$ peak, whereas a slight trend could be suggested for the $2110 \mathrm{~cm}^{-1}$ one. Therefore, the higher intensities of the two latter peaks observable in the 488-nm spectrum might be linked to the presence of the above-mentioned background fluorescence. Nonetheless, a resonant process can probably be assumed also in this case, certainly for the $2110 \mathrm{~cm}^{-1}$ band.

\section{B. Temperature dependence of $\mathrm{PuO}_{2}$ Raman bands}

SAS Raman spectra obtained with the 514-nm excitation for various laser power levels are shown in Fig. 2. Prior to the Stokes and anti-Stokes spectra collection, the sample was irradiated for several minutes with a high laser power (about $1 \mathrm{~W}$ at the exit of the cavity), in order to locally anneal in situ possible self-radiation damage effects in the investigated spot. The excitation source yielding the best accuracy for the current Stokes and anti-Stokes (SAS) Raman analysis was the 514-nm laser line. Lower energy excitation sources were observed to result, even at the highest power levels, in a very limited sample heating and in any case in a low signal-to-noise ratio in the anti-Stokes spectrum. On the other hand, a nonnegligible fluorescence background, clearly observable also in Fig. 1, would affect the analysis in spectra recorded around the 488-nm laser line.

Figures 3(a)-3(c) display the resulting temperature evolution of the spectral peak position $\Delta \omega / \omega_{0}=$ $\frac{\omega(T)-\omega_{298} \mathrm{~K}}{\omega_{298}}$ full width at half maximum FWHM $\frac{\Delta \Omega}{\Omega_{0}}=\frac{\Omega(\mathrm{T})-\Omega_{298 \mathrm{~K}}}{\Omega_{298 \mathrm{~K}}}$ and intensity nnormalized as $\left.I\left(T_{2 g}\right)=I\left(T_{2 g}\right) /\left[I\left(T_{2 g}\right)+I(2110)+I(2620)\right]\right\}$ for the three main Raman modes observed in the current spectra, the $T_{2 g}$ peak, and the two higher energy peaks centered at 2110 and $2620 \mathrm{~cm}^{-1}$. These bands were fitted, after baseline correction, with unconstrained Lorentzian curves. 


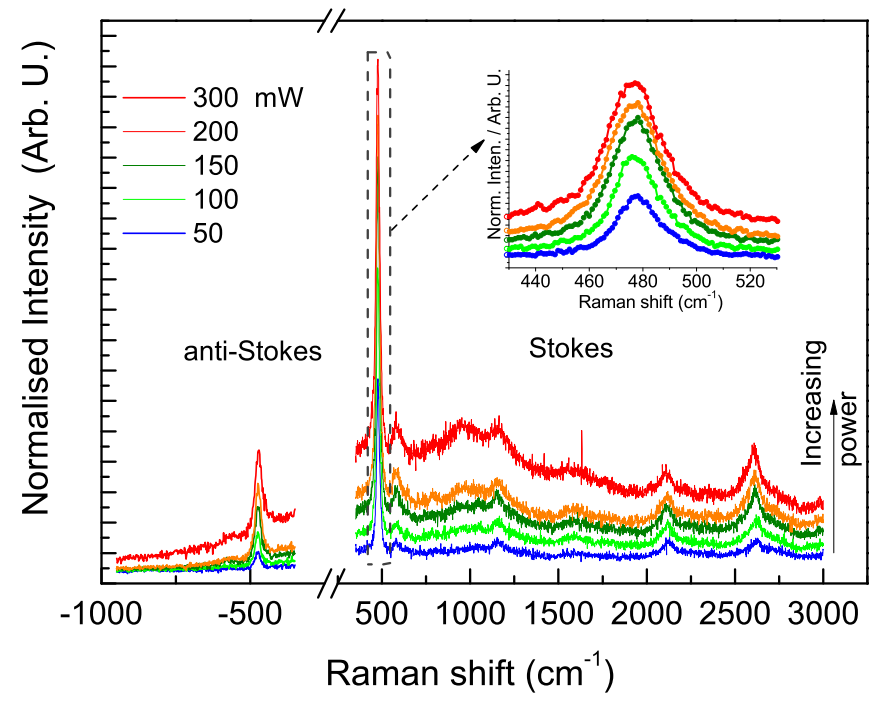

FIG. 2. Stokes and anti-Stokes Raman spectra obtained with $514 \mathrm{~nm}(2.41 \mathrm{eV})$ for different laser power. The solid-line arrow shows the increasing power from 50 to $300 \mathrm{~mW}$. The inset shows the $T_{2 g}$ peak shift with increasing the laser power. Laser power is recorded at the sample surface.

The temperature dependence of peak positions of the two high energy modes at 2110 and $2620 \mathrm{~cm}^{-1}$, respectively, decreases at increasing temperatures, much more than the $T_{2 g}$ band. Correspondingly, a much larger value of the Grüneisen parameter can be obtained for both high-energy peaks, in both cases between 15 and 20. Such a high value of $\gamma$ is probably meaningless, if the bands under investigation are to be considered as purely vibrational modes [10]. Such a strong peak shift was frequently observed for modes involving electronic excitations [33-35]. In this case, if one considers that the high-energy bands have an electronic origin, then the peak shift is due to the oscillations around the equilibrium position of the $\mathrm{Pu}^{4+}$ ion, on which the electronic transition occurs, and to those of the surrounding ions (lattice vibrations). As the temperature increases a lattice thermal expansion takes place, softening, as a rule, the interaction between the central ion and the surroundings; as a consequence, a downshift is expected.

As shown in Figs. 1 and 2, the peak width of these highenergy modes is much larger (FWHM $\sim 20 \mathrm{~cm}^{-1}$ ) than the one observed for the $T_{2 g}$ mode. For ordered systems such high FWHM values are meaningless if the modes have a vibrational origin. In fact, it is possible that at these temperatures phonon scattering has already largely reduced the lifetime of these high-energy excitations. Fast electronic relaxation occurring at these temperatures $(T \sim 298 \mathrm{~K})$ were already observed in similar materials like $\mathrm{CeB}_{6}$ and $\mathrm{Pr}_{2} \mathrm{CuO}_{4}$ [34,36]. Assuming that these peaks rise from electronic excitations, the peak width is then tightly connected with the local environment probed by $\mathrm{Pu}^{4+}$. The $T$-independent trend of both high-energy modes, as portrayed in Fig. 3(b), suggests that $\mathrm{Pu}^{4+}$ crystal field does not experience a strong change in the local environment with temperature, mainly because of the shield provided by the outermost electrons. For temperatures below $2000 \mathrm{~K}$, Hutchings [37] has shown that $\mathrm{UO}_{2}$ and $\mathrm{ThO}_{2}$ do not exhibit any

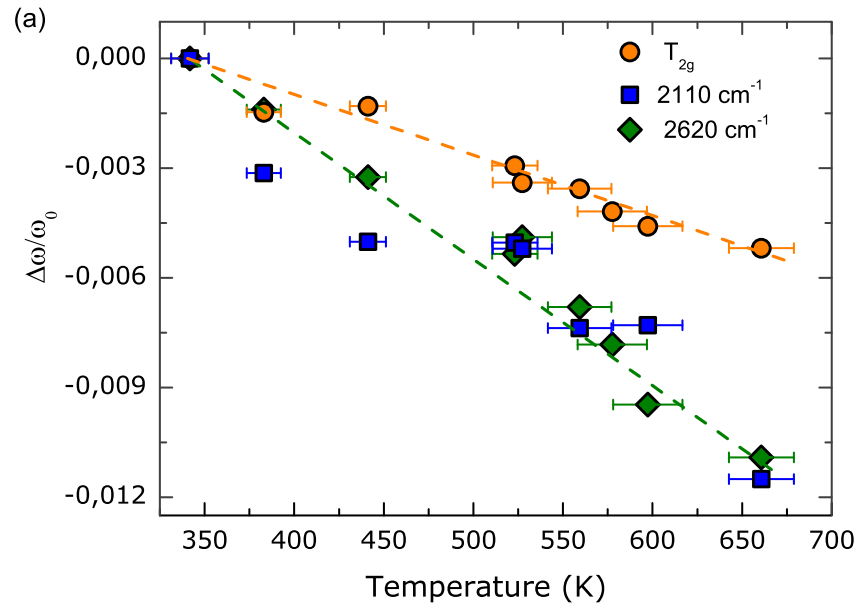

(b)
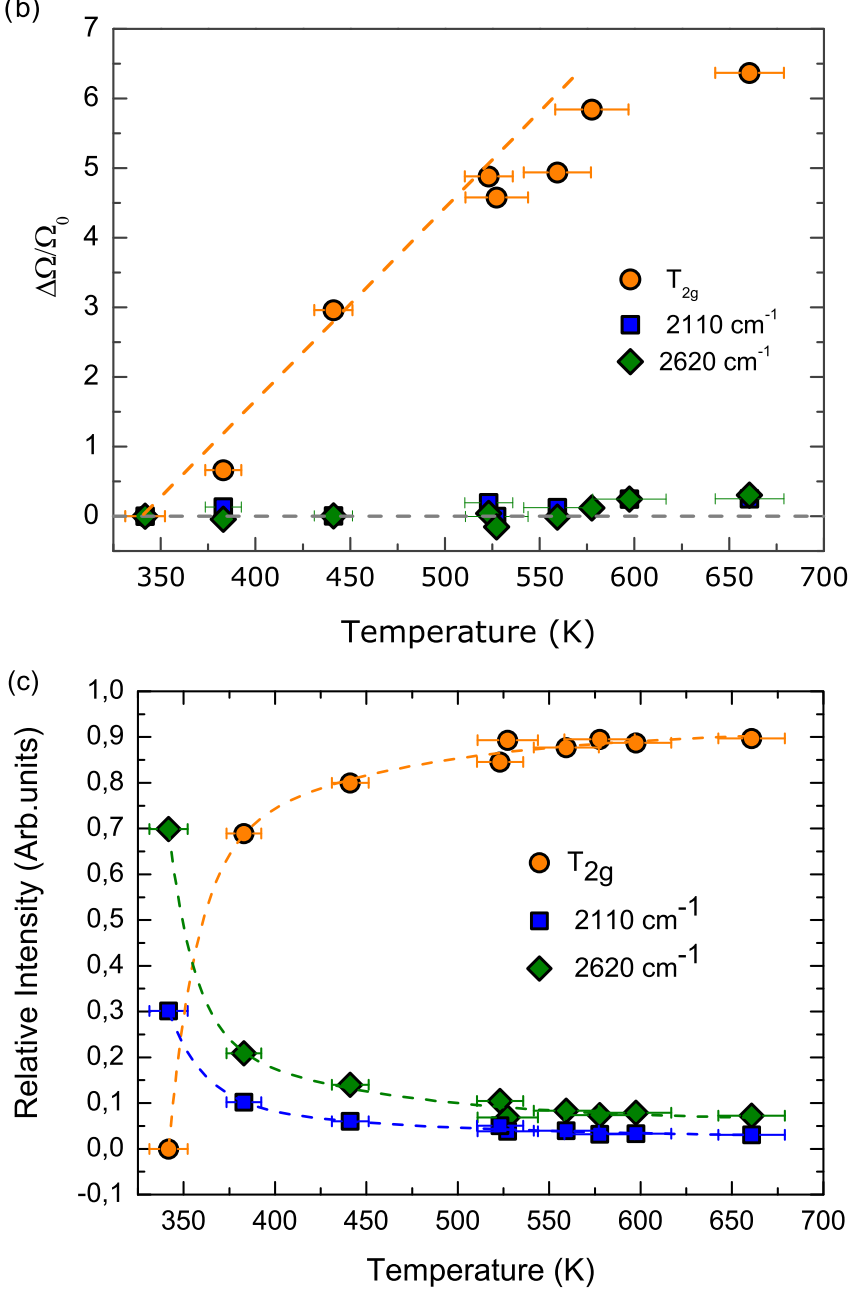

FIG. 3. (a) Temperature dependence of peak shift $\Delta \omega / \omega_{0}=$ $\frac{\omega(T)-\omega_{298} \mathrm{~K}}{\omega_{298}}$, (b) FWHM $\frac{\Delta \Omega}{\Omega_{0}}=\frac{\Omega(\mathrm{T})-\Omega_{298} \mathrm{~K}}{\Omega_{298 \mathrm{~K}}}$, and (c) relative intensity $I\left(T_{2 g}^{\omega_{2}}\right)=I\left(T_{2 g}\right) /\left[I\left(T_{2 g}\right)+I(2110)+I(2620)\right]$ of $T_{2 g}$ (filled circles), $2110 \mathrm{~cm}^{-1}$ (filled squares), and $2620 \mathrm{~cm}^{-1}$ (filled diamonds) bands. Dashed lines in (a) represent a linear fit of the peak position, and in (b) and (c) are guides to the eye.

dynamical disorder, which further support the attribution of these modes to electronic excitations. Furthermore, Mansouri et al. [38] has shown that in $\mathrm{TbMn}_{2} \mathrm{O}_{5}$ and $\mathrm{HoMn}_{2} \mathrm{O}_{5}$ the peak 
TABLE II. Comparison between the calculated and measured CF energy splitting (expressed in $\mathrm{cm}^{-1}$ ) for the lowest manifold of PuO $\mathrm{O}_{2}$, from the present work as well as literature sources. The energy of the lowest level $\left(\Gamma_{1}\right)$ has been set to zero in all cases.

\begin{tabular}{|c|c|c|c|c|c|c|c|c|c|}
\hline \multirow[b]{2}{*}{ irrep } & \multicolumn{7}{|c|}{ Calculated energies } & \multicolumn{2}{|c|}{ Measured energies } \\
\hline & Ref. [16] & Ref. [39] & Ref. [40] & Ref. [42] & Ref. [43] & Ref. [44] & This work & Ref. [40] & This work \\
\hline$\Gamma_{4}$ & 1081 & 929 & 928 & 799 & 518 & 782 & 1055 & 1000 & - \\
\hline$\Gamma_{5}$ & 2113 & 1776 & - & 1678 & 1024 & 1645 & 2153 & - & 2110 \\
\hline$\Gamma_{3}$ & 2710 & 2153 & - & 1307 & 829 & 1573 & 2630 & - & 2620 \\
\hline
\end{tabular}

width of CF excitations is highly affected by the dynamic of the $\mathrm{R}-\mathrm{O}$ bonds $(\mathrm{R}=\mathrm{Tb}, \mathrm{Ho})$.

Regarding the temperature dependence of the intensity of the bands, it is important to note that in the absence of an absorption spectrum of $\mathrm{PuO}_{2}$ it is rather difficult to draw strong conclusions on the temperature behavior of the modes, as many factors may add/cancel to each other. However, one can still draw a few conclusions regarding the relative intensity evolution of the vibrational and high-energy modes (below $450 \mathrm{~K})$. The relative intensity of the $T_{2 g}$ mode increases, while the temperature dependence of the peaks at 2110 and $2620 \mathrm{~cm}^{-1}$ is essentially different; it decreases at the beginning of the investigated temperature range, up to approximately $400 \mathrm{~K}$.

Thus, the markedly different temperature dependence of both modes, when compared with the purely vibrational $T_{2 g}$ line, proves that these two peaks are of a different nature as already suggested by Sarsfield et al. [12]. In order to complete the assignment of these modes, in this work $\mathrm{PuO}_{2}$ $\mathrm{CF}$ energy levels have been recalculated with an updated set of parameters.

\section{CRYSTAL FIELD CALCULATIONS}

Krupa and Gajek [39] exploited $a b$ initio calculations in order to establish the tendency of the evolution of the CF potential as a function of the atomic number for the actinide dioxide series, thus obtaining a set of parameters for $\mathrm{PuO}_{2}$ from experimental spectroscopic studies of the isostructural $\mathrm{UO}_{2}$ and $\mathrm{NpO}_{2}$. In a similar fashion, Kern et al. [40] used Newman's superposition model [41] to determine how the $\mathrm{UO}_{2} \mathrm{CF}$ parameters would rescale to $\mathrm{PuO}_{2}$. In both cases, the calculated position of the $\Gamma_{4}$ triplet is about $930 \mathrm{~cm}^{-1}$ above the $\Gamma_{1}$ ground singlet; this is in good agreement with inelastic neutron scattering (INS) experiments on polycrystalline ${ }^{242} \mathrm{PuO}_{2}$, which have revealed a single wide peak around $1000 \mathrm{~cm}^{-1}$ that must be assigned to the intramultiplet $\Gamma_{1} \rightarrow \Gamma_{4}$ transition (the only one allowed by the magnetic-dipole selection rules which govern INS) [42]. The parameter set used in the latter paper also predicts correctly the absolute value of the neutron cross section.

This interpretation leaves one problem open: the energy gap value measured by INS is way smaller than the lower bound estimated from the measured magnetic susceptibility [14]. For this reason, Colarieti-Tosti et al. [42] performed a LDA ab initio study of the electronic structure of $\mathrm{PuO}_{2}$ and included antiferromagnetic exchange enhancement; however, this is not sufficient to completely remove the discrepancy. The CF splittings that they calculate are somewhat smaller with respect to the experimental data, which is also true for other, more recent $a b$ initio studies [43,44]. All of these papers also predict that the order of the $\Gamma_{3}$ and $\Gamma_{5}$ excited levels belonging to the ground ${ }^{5} I_{4}$ manifold is reversed with respect to Krupa and Gajek's results [39]; we attribute this to the relatively close position of the $\Gamma_{5}$ triplet belonging to the excited ${ }^{5} I_{5}$ manifold (at an energy around $5000 \mathrm{~cm}^{-1}$ ), which mixes with the lower ${ }^{5} I_{4}-\Gamma_{5}$ triplet and pushes it down in energy when the CF is stronger. This consideration emphasizes the need to correctly estimate the CF parameters and, in particular, to fully account for $J$ mixing in the calculations [45].

The failure to reconcile the existing bulk and spectroscopic measurements for $\mathrm{PuO}_{2}$ is an extremely complicated problem to solve, to the point that first-principle calculations even questioned the validity of the single-ion approximation itself in describing the electronic ground state of $\mathrm{PuO}_{2}$ [46]. Conversely, recent CASSCF calculations suggested that the observed temperature-independent paramagnetism arises from a competition between the different excited electronic states [16]. In this section we show that calculations performed with the most reliable values of the CF parameters reproduce the spectroscopically determined energy splitting of the lowenergy manifold very well and are perfectly consistent with an electronic attribution of the two Raman peaks detected at 2110 and $2620 \mathrm{~cm}^{-1}$. The appropriate single-ion Hamiltonian for a tetravalent $\mathrm{Pu}$ ion in a CF potential of cubic symmetry is

$$
\begin{aligned}
H= & \sum_{k=2,4,6} F^{k} f_{k}+\zeta_{5 f} \sum_{n=1}^{4} \mathbf{s}_{n} \cdot \mathbf{l}_{n}+\alpha G\left(R_{3}\right)+\beta G\left(G_{2}\right) \\
& +\gamma G\left(R_{7}\right)+\sum_{i=2,3,4,6,7,8} T^{i} t_{i}+\sum_{j=0,2,4} M^{j} m_{j} \\
& +\sum_{k=2,4,6} P^{k} p_{k}+B_{4}\left[C_{0}^{(4)}+\sqrt{\frac{5}{14}}\left(C_{+4}^{(4)}+C_{-4}^{(4)}\right)\right] \\
& +B_{6}\left[C_{0}^{(6)}-\sqrt{\frac{7}{2}}\left(C_{+4}^{(6)}+C_{-4}^{(6)}\right)\right]
\end{aligned}
$$

where $F^{2}, F^{4}$, and $F^{6}$ are the Slater radial integrals quantifying the nonspherical part of the electronic repulsion, $\zeta_{5 f}$ is the spin-orbit coupling constant for the $5 f$ shell, $\alpha, \beta$, and $\gamma$ are the Trees parameters associated with two-body operators which perturbatively account for multiconfigurational effects, the $T^{i}$ parameters are related to effective operators which describe three-body interactions, the Marvin parameters $M^{j}$ account for further relativistic corrections such as spin-spin and spin-otherorbit coupling, $P^{k}$ represent the electrostatically correlated magnetic interactions, and finally $B_{4}$ and $B_{6}$ quantify the cubic 
CF potential [41]. For the reasons stated above, we consider that the best estimate for the two latter parameters of $\mathrm{PuO}_{2}$ is that given by Kern et al. [40] as $B_{4}=-9760 \mathrm{~cm}^{-1}$ and $B_{6}=+3968 \mathrm{~cm}^{-1}$. All other parameters which appear in $H$ are related to free-ion interactions and, therefore, practically independent of the host matrix; for this reason, we have fixed their values to those determined by Carnall et al. [47] for tetravalent $\mathrm{Pu}$ ions in $\mathrm{PuF}_{4}$.

The energy levels for the lowest manifold calculated by a full diagonalization of $H$ (in order to correctly take both intermediate coupling and $J$ mixing into account) are reported in Table II, together with the irreducible representation (irrep) of each level (as for the degeneracy of electronic multiplets, we recall that the $\Gamma_{1}$ state is a singlet, $\Gamma_{3}$ is a doublet, and $\Gamma_{4}$ and $\Gamma_{5}$ are triplets). Experimental spectroscopic data are now available for the whole manifold $\left({ }^{5} I_{4}\right)$; focusing on that, it can be noticed that not only the energy experimentally measured by INS for the $\Gamma_{1} \rightarrow \Gamma_{4}$ transition is correctly reproduced by the single-ion model, but also that the predicted $\Gamma_{1} \rightarrow \Gamma_{5}$ and $\Gamma_{1} \rightarrow \Gamma_{3}$ electronic transition energies are in very good quantitative agreement with the position of the Raman peaks measured in this work. Remarkably, our results are also in agreement with the CASSCF calculations performed by Gendron and Autschbach [16]. Both latter transitions are allowed by the quadrupolar selection rules, which justify their presence in our unpolarized Raman measurement. This correlation clarifies the origin of the as yet unassigned features in the Raman spectrum and completes the general picture of the CF levels within ${ }^{5} I_{4}$. Since over this energy range, only pure $\mathrm{CF}$ excitations are expected and no phonon or multiphonon process is expected, as compared to $\mathrm{UO}_{2}$, both bands should be exclusively attributed to pure $\mathrm{CF}$ excitations.

Importantly, these modes were observed also for $\mathrm{PuO}_{2}-\mathrm{ThO}_{2}$ and $\mathrm{PuO}_{2}-\mathrm{UO}_{2}$ solid solutions [6,48], and their intensity increases with the increase of the plutonium content. This feature is very helpful to identify $\mathrm{PuO}_{2}$ when mixed or dissolved in other compounds, since the vibrational $T_{2 g}$ mode usually overlap with other active vibrations in the phonon spectral region.

Finally, it is interesting to note that the frequency of the mode at $956 \mathrm{~cm}^{-1}$ coincides with the $\Gamma_{1} \rightarrow \Gamma_{4}(\mathrm{CF})$ transition, as observed by INS experiment and predicted by DFT and CF calculations $[39,40,42]$. The overlapping of this band with the 2LO2 mode especially at high temperatures makes the analysis of its temperature dependence rather difficult and its assignment to a pure 2TO2, 2LO1 mode or $\Gamma_{1} \rightarrow \Gamma_{4} \mathrm{Pu}^{4+} \mathrm{CF}$ excitation ambiguous.

\section{CONCLUSIONS}

In the present investigation we have described and discussed the main features of the Raman spectrum of plutonium dioxide. The characteristics of the peaks recorded at 2110 and $2620 \mathrm{~cm}^{-1}$ have been broadly discussed in relation to their temperature dependence. Their most plausible attribution is an electronic nature. In particular, their spectral positions match well the $\Gamma_{1} \rightarrow \Gamma_{5}$ and $\Gamma_{1} \rightarrow \Gamma_{3}$ crystal electric field transitions calculated in this work. This correlation clarifies the origin of the as yet unassigned features in the Raman spectrum and completes the general picture of the CF levels within the ${ }^{5} I_{4}$ manifold. The reported peaks at 2110 and $2620 \mathrm{~cm}^{-1}$ and their attributions define a peculiar Raman fingerprint of plutonium dioxide, which can be used for the in situ and ex situ detection of this important compound for nuclear safety and safeguard. As a closing remark, we reiterate that the single-ion model can naturally describe all the experimental spectroscopic features of $\mathrm{PuO}_{2}$ within the same framework that works well for the whole actinide dioxide series [45]; this will have to be taken into account in future attempt to theoretically describe its electronic ground state [49].

\section{ACKNOWLEDGMENTS}

M.N. would like to acknowledge the European Commission for the financial support. S.M. acknowledges the EURATOM FP7 project GENTLE (Contract No. 323304) for supporting her stay at JRC Karlsruhe. The authors would like to thank Prof. R. Caciuffo and Prof. R. Konings (JRC, ITU) for reading our manuscript. We also would like to thank Dr. P. Simon (CEMHTI-CNRS) and Dr. D. Neuville (IPGP, CNRS) for their fruitful discussions.
[1] D. M. L. Ho, D. Manara, P. Lindqvist-Reis, T. Fanghänel, and K. Mayer, Vib. Spectrosc. 73, 102 (2014).

[2] D. M. L. Ho, A. E. Jones, J. Y. Goulermas, P. Turner, Z. Varga, L. Fongaro, T. Fanghänel, and K. Mayer, Foren. Sci. Int. 251, 61 (2015).

[3] C. Jégou, M. Gennisson, S. Peuget, L. Desgranges, G. Guimbretière, M. Magnin, Z. Talip, and P. Simon, J. Nucl. Mater. 458, 343 (2015).

[4] C. Jégou, R. Caraballo, S. Peuget, D. Roudil, L. Desgranges, and M. Magnin, J. Nucl. Mater. 405, 235 (2010).

[5] J. R. Schoonover, F. Weesner, G. J. Havrilla, M. Sparrow, and P. Treado, Appl. Spectrosc. 52, 1505 (1998).

[6] R. Böhler et al., J. Nucl. Mater. 448, 330 (2014).

[7] T. Shimanouchi, M. Tsuboi, and T. Miyazawa, J. Chem. Phys. 35, 1597 (1961).
[8] G. M. Begun, R. G. Haire, W. R. Wilmarth, and J. R. Peterson, J. Less Common Metals 162, 129 (1990).

[9] R. Böhler et al., J. Alloys Compd. 616, 5 (2014).

[10] T. Livneh and E. Sterer, Phys. Rev. B 73, 085118 (2006).

[11] R. Rao, R. K. Bhagat, N. P. Salke, and A. Kumar, Appl. Spectrosc. 68, 44 (2014).

[12] M. J. Sarsfield, R. J. Taylor, C. Puxley, and H. M. Steele, J. Nucl. Mater. 427, 333 (2012).

[13] P. Santini, S. Carretta, G. Amoretti, R. Caciuffo, N. Magnani, and G. H. Lander, Rev. Mod. Phys. 81, 807 (2009).

[14] G. Raphael and R. Lallement, Solid State Commun. 6, 383 (1968).

[15] N. M. Edelstein and G. H. Lander, The Chemistry of the Actinide and Transactinide Elements (Springer, Berlin, 2008), p. 2225 . 
[16] F. Gendron and J. Autschbach, J. Phys. Chem. Lett. 8(3), 673 (2017).

[17] J. Koningstein and C. Kane-Maguire, Can. J. Chem. 52, 3445 (1974).

[18] L. Tsachi, J. Phys.: Condens. Matter 20, 085202 (2008).

[19] M. Naji, J.-Y. Colle, O. Beneš, M. Sierig, J. Rautio, P. Lajarge, and D. Manara, J. Raman Spectrosc. 46, 750 (2015).

[20] F. De Bruycker, K. Boboridis, P. Pöml, R. Eloirdi, R. Konings, and D. Manara, J. Nucl. Mater. 416, 166 (2011).

[21] E. Gardner, T. Markin, and R. Street, J. Inorgan. Nucl. Chem. 27, 541 (1965).

[22] H. Richter, Z. Wang, and L. Ley, Solid State Commun. 39, 625 (1981).

[23] M. Naji, F. Di Lemma, A. Kovács, O. Beneš, D. Manara, J. Y. Colle, G. Pagliosa, P. Raison, and R. J. M. Konings, J. Raman Spectrosc. 46, 661 (2015).

[24] M. Naji, N. Magnani, J. Y. Colle, O. Beneš, S. Stohr, R. Caciuffo, R. J. M. Konings, and D. Manara, J. Phys. Chem. C 120, 4799 (2016).

[25] F. LaPlant, G. Laurence, and D. Ben-Amotz, Appl. Spectrosc. 50, 1034 (1996).

[26] P. Zhang, B.-T. Wang, and X.-G. Zhao, Phys. Rev. B 82, 144110 (2010).

[27] Q. Yin and S. Y. Savrasov, Phys. Rev. Lett. 100, 225504 (2008).

[28] L. Desgranges, G. Guimbretière, P. Simon, C. Jegou, and R. Caraballo, Nucl. Instrum. Methods Phys. Res. Sect. B 315, 169 (2013).

[29] L. Desgranges, G. Baldinozzi, P. Simon, G. Guimbretière, and A. Canizares, J. Raman Spectrosc. 43, 455 (2012).

[30] D. Manara and B. Renker, J. Nucl. Mater. 321, 233 (2003).

[31] K. Nakamoto, Infrared and Raman Spectra of Inorganic and Coordination Compounds (Wiley Online Library, New York, 1986).

[32] A. C. Ferrari and D. M. Basko, Nat. Nano 8, 235 (2013).
[33] R. Wegerer, C. Thomsen, T. Ruf, E. Schönherr, M. Cardona, M. Reedyk, J. S. Xue, J. E. Greedan, and A. Furrer, Phys. Rev. B 48, 6413 (1993).

[34] J. A. Sanjurjo, C. Rettori, S. Oseroff, and Z. Fisk, Phys. Rev. B 49, 4391(R) (1994).

[35] G. Güntherodt, A. Jayaraman, G. Batlogg, M. Croft, and E. Melczer, Phys. Rev. Lett. 51, 2330 (1983).

[36] E. Zirngiebl, B. Hillebrands, S. Blumenröder, G. Güntherodt, M. Loewenhaupt, J. M. Carpenter, K. Winzer, and Z. Fisk, Phys. Rev. B 30, 4052(R) (1984).

[37] M. T. Hutchings, J. Chem. Soc. Faraday Trans. 2 83, 1083 (1987).

[38] S. Mansouri, S. Jandl, M. Balli, J. Laverdière, P. Fournier, and D. Z. Dimitrov, Phys. Rev. B 94, 115109 (2016).

[39] J. Krupa and Z. Gajek, Eur. J. Solid State Inorg. Chem. 28, 143 (1991).

[40] S. Kern, R. A. Robinson, H. Nakotte, G. H. Lander, B. Cort, P. Watson, and F. A. Vigil, Phys. Rev. B 59, 104 (1999).

[41] D. J. Newman and B. Ng, Crystal Field Handbook (Cambridge University Press, Cambridge, 2000).

[42] M. Colarieti-Tosti, O. Eriksson, L. Nordström, J. Wills, and M. S. S. Brooks, Phys. Rev. B 65, 195102 (2002).

[43] G. Gaigalas, E. Gaidamauskas, Z. Rudzikas, N. Magnani, and R. Caciuffo, Lithuanian J. Phys. 49, 403 (2009).

[44] F. Zhou and V. Ozoliņš, Phys. Rev. B 85, 075124 (2012).

[45] N. Magnani, P. Santini, G. Amoretti, and R. Caciuffo, Phys. Rev. B 71, 054405 (2005).

[46] A. B. Shick, J. Kolorenč, L. Havela, T. Gouder, and R. Caciuffo, Phys. Rev. B 89, 041109(R) (2014).

[47] W. Carnall, G. Liu, C. Williams, and M. Reid, J. Chem. Phys. 95, 7194 (1991).

[48] R. Böhler, P. Çakır, O. Beneš, H. Hein, R. J. M. Konings, and D. Manara, J. Chem. Thermodyn. 81, 245 (2015).

[49] M. T. Suzuki, N. Magnani, and P. M. Oppeneer, Phys. Rev. B 88, 195146 (2013). 